\title{
Impacts des changements de l'occupation du sol et des changements climatiques sur le bassin versant de la rivière Davo, Côte d'Ivoire
}

\author{
Touao Kah Martine Gauze, \\ Centre de Recherche en Ecologie, Abidjan, Côte d'Ivoire \\ Kouamé Yao Morton, \\ Laboratoire Géoscience et Environnement, \\ Université Nangui Abrogoua, Abidjan, Côte d'Ivoire \\ Mélèdje N'diaye Hermann, \\ Sékongo Guénolé Largaton, \\ Centre de Recherche en Ecologie, Abidjan, Côte d'Ivoire \\ Soro Gnéneyougo Emile, \\ Laboratoire Géoscience et Environnement, \\ Université Nangui Abrogoua, Abidjan, Côte d'Ivoire
}

Doi: 10.19044/esj.2018.v14n33p408 URL:http://dx.doi.org/10.19044/esj.2018.v14n33p408

\begin{abstract}
The main objective of this study is to asssess the impacts of climate change and land cover change on the hydrology of the Davo river basin located in southwestern of Côte d'Ivoire. Two scenarios of climate change (A2 and B2) and two scenarios of land cover called scenario 1 (deforestation) and scenario 2 (reforestation) were established, and streamflows under these scenarios were simulated by the hydrological model CEQUEAU. Each scenario showed distinct variations in streamflows. Regarding the impacts of land cover change, scenario 1 and scenario 2 indicate increases of $10.6 \%$ and $1 \%$, respectively. As for the impacts of climate change, climate models predict a very wide range of possible changes in streamflows. The multimodel approach indicates a decrease in streamflows of $0.5 \%$ and $2.75 \%$ respectively under the A2 and B2 scenarios by 2050. By 2080, streamflows would increase by $14.75 \%$ under the scenario A2 and $3.5 \%$ under scenario B2. In order to implement adaptation and mitigation strategies, Representative Concentration Pathways (RCP) scenarios should be used, regional climate models should be applied, and combined assessments of the impacts of climate change and land cover should be made.
\end{abstract}


Keywords: Land cover change, climate change, hydrology impact, Davo river basin, CEQUEAU

\section{Résumé}

L’objectif principal de la présente étude est d'évaluer les impacts des changements climatiques et des changements d'occupation du sol sur l'hydrologie du bassin versant de la Davo.situé au Sud-Ouest de la Côte d'Ivoire. Deux scenarios des changements climatiques (A2 et B2) et deux des changements d'occupation du sol dénommés scenarios 1 (déforestation) et scénario 2 (reforestation) d'occupation du sol ont été établis, et les débits sous ces scenarios ont été simulés par le modèle hydrologique CEQUEAU. Chaque scenario a montré des variations distinctes de débits. Concernant les impacts des changements d'occupation du sol, le scenario 1 et le scenario 2 indiquent respectivement des augmentations $10,6 \%$ et $1 \%$ des débits. Quant aux impacts des changements climatiques, les modèles climatiques prédisent une gamme très large des évolutions possibles des débits. L'approche multimodèle indique une diminution des débits de $0,5 \%$ et de $2,75 \%$ respectivement sous les scenarios A2 et B2 à l'horizon 2050. A 1'horizon 2080, les débits augmenteraient de $14,75 \%$ sous le scenario A2 et de 3,5\% sous le scenario B2. Dans une perspective de mettre en place des stratégies d'adaptation et d'atténuation, il convient d'utiliser des scénarios RCP (Representative Concentration Pathways), d'appliquer les modèles climatiques régionaux et faire l'évaluation combinée des impacts des changements climatiques et des changements d'occupation du sol.

Mots clés: Changements d'occupation du sol, changements climatiques, impacts hydrologiques, bassin versant de davo, CEQUEAU

\section{Introduction}

Les changements climatiques et les changements d'occupation du sol sont reconnus comme les principaux problèmes environnementaux globaux (Pielke, 2005 ; Boyd et al., 2008). Ils sont susceptibles d'affecter les ressources en eau dans les bassins versants (Praskievicz et Chang, 2011). L'Afrique et en particulier l'Afrique subsaharienne fait face à ces nouveaux défis. Selon Cornelissen et al., (2013), les changements climatiques et les changements de l'occupation du sol ont déjà des impacts sur le cycle hydrologique dans les pays en Afrique de l'ouest. En côte d'Ivoire, les résultats des études menées sur les fluctuations climatiques dans certaines zones de la Côte d'Ivoire montrent la baisse de la pluviométrie (Savané et al., 2001 ; Goula et al., 2006 ; Kouakou et al., 2007, Yao et al., 2012). De même, les études menées sur certains bassins versants ont montré également une baisse des ressources en eau de surface et souterraine (Savané et al., 2001 ; 
Goula et al., 2006 ; Kouakou et al., 2007 ; Goula et al., 2009 ; Yao et al., 2012). Kouakou et al. (2012) ont caractérisé une hausse des températures dans les études menées sur toute la Côte d'Ivoire. Au-delà de la baisse de la pluviométrie et des ressources en eau, et de la hausse des températures, la Côte d'Ivoire connait une régression de son couvert forestier du à l'augmentation des zones agricoles (Brou 2010. Cette diminution des ressources forestières a pour conséquences la baisse de la pluviométrie (Brou 2005). Du fait de l'étroite corrélation entre les variations hydroclimatiques et la modification du couvert végétal, la prise en compte des changements d'occupation du sol dans les études d'impacts des changements climatiques sur les ressources en eau, est indispensable. De nombreuses études ont quantifié la quantité d'eau sous les changements climatiques et les changements d'occupation du sol séparément (Metzger et al., 2008 ; Montenegro et Ragad, 2012; Fan et Shibata, 2015; Neupane et al., 2015). Cependant, quelques études ont employé l'approche combinée c'est-à-dire en analysant et en comparant les impacts des changements climatiques et des changements d'occupation du sol sur la même période (Chiang et al., 2012 ; Kim et al., 2013, Neupane et al., 2015). La présente étude a pour objectif d'évaluer séparément les impacts potentiels des changements climatiques et des changements d'occupation du sol sur l'hydrologie du bassin versant de la rivière Davo situé au Sud-ouest de la Côte d'Ivoire. Cette zone subit des pressions anthropiques excessives. La rapidité et l'ampleur des transformations paysagères font de cette région un espace privilégié pour étudier les interactions entre le climat et l'occupation du sol.

\section{Matériels et méthodes Site d'étude}

Le bassin versant de la rivière Davo situé au Sud-ouest de la Côte d'Ivoire. Il s'étend de la longitude $6^{\circ} 47 \mathrm{~W}$ à longitude $5^{\circ} 69 \mathrm{~W}$ et de la latitude $6^{\circ} 85 \mathrm{~N}$ à la latitude $5^{\circ} 03 \mathrm{~N}$. Il couvre une superficie de $7025 \mathrm{~km}^{2}$ (figure 1). C'est une zone qui fait partie de l'économie café-cacao de la Côte d'Ivoire (Touao, 1993). De l'aval à l'amont du bassin versant, les altitudes rencontrées sont comprises entre $34 \mathrm{~m}$ et $300 \mathrm{~m}$ pour près de $90 \%$ de sa superficie totale. Le climat est de type tropical humide. Les précipitations sont variables d'une année à l'autre avec une pluie moyenne annuelle de l'ordre de $1433 \mathrm{~mm}$. La température moyenne annuelle est de $26^{\circ} \mathrm{C}$. 


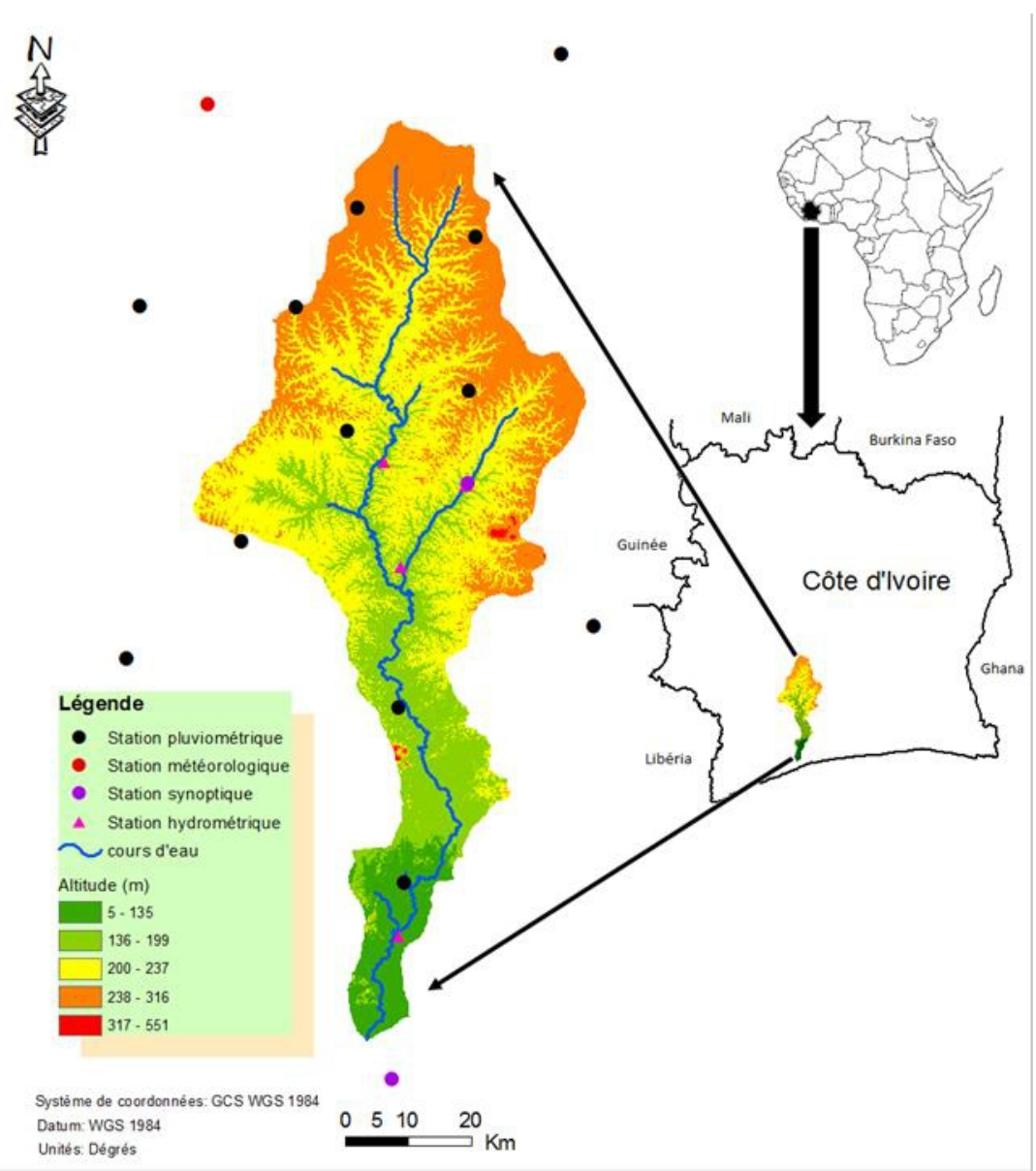

Figure 1. Localisation du bassin versant de la Davo.

\section{Données et méthodes}

\section{Données de pluie, de température et de débit}

Les données météorologiques utilisées dans cette étude sont issues de la Société d'Exploitation et de Développement Aéroportuaire, Aéronautique et Météorologique (SODEXAM). Les stations sélectionnées sont au nombre de quinze. Elles sont régulières et homogènes aux stations synoptiques et météorologiques et comportent des lacunes aux stations pluviométriques. Huit stations ont servi au fonctionnement du modèle hydrologique. Les autres stations ont permis de combler certaines données. Les données hydrométriques sont issues de la base de données de la Direction de l'hydraulique Humaine du Ministère des Infrastructures économiques. Le bassin dispose de quatre stations hydrométriques. Elles comportent des lacunes, mais sont peu régulières à la station de Dapkadou qui a servi au calage et à la validation du modèle hydrologique. Les informations sur l'occupation du sol pour les années 1986 et 2014 ont été obtenues des travaux de Kouamé 
(2017). Quatre classes majeures d'occupation du sol ont été définies (forêt, agriculture et jachère, zones bâties et sols, eau).

\section{Scénarios des changements climatiques et des changements d'occupation du sol}

Les précipitations et les températures simulées par les modèles climatiques pour la période de référence (1980-1999) et les périodes futures (2040-2069 et 2070-2099) ont été obtenues du générateur des scénarios climatiques MAGICC/SCENGEN (Kouamé et al., 2014). Les données des modèles climatiques globaux ECHAM5, GFDL, CSIRO, HadCM3 (tableau 1) contenues dans MAGICC SCNEGEN (Wigley, 2008) ont été utilisées. Deux scénarios d'émission de gaz à effet de serre ont été considérés. Ces deux scénarios étaient $\mathrm{A} 2$ et $\mathrm{B} 2$. A2 et $\mathrm{B} 2$ décrivent un développement hétérogène. A2 met l'accent sur un développement économique sur le schéma actuel et B2 met l'accent sur un développement soucieux de l'environnement et du développement durable.

Tableau 1. Modèles Climatiques Globaux retenus pour les projections climatiques (Kouamé et al, 2014).

\begin{tabular}{ccc}
\hline Nom du MCG & $\begin{array}{c}\text { Institution } \\
\text { CSIRO_MK3.0 }\end{array}$ & Résolution \\
Commonwealth Scientific and Industrial ResearchOrganization & (Australie) & $2.8^{\circ} \times 2.8^{\circ}$ \\
MPI_ECHAM5 & Max Planck Institute for Meteorology (Allemagne) & $1.9^{\circ} \times 1.9^{\circ}$ \\
GFDL_CM2.1 & NOAA Geophysical Fluid Dynamics Laboratory (Etats-Unis) & $2.5^{\circ} \times 2.0^{\circ}$ \\
UKMO-HadCM3 & Hadley Center for Climate Prediction and Research Met Office & $2.5^{\circ} \times 3.75^{\circ}$ \\
\hline
\end{tabular}

Deux scénarios d'occupation du sol (scénario 1 et scénario 2) réalisés par Kouamé (2017) avec le modèle de prédiction de l'occupation du sol Land Change Modeler ont été utilisés (figure 2, tableau 2) dans cette étude. Le modèle Land Change Modeler a été développé par le Clark Lab de l'Université de Worcester aux Etats-Unis (Eastman, 2015). Dans cette étude, le scénario 1 est considéré comme la déforestation dans lequel il n'y a pas de conservation des ressources naturelles (forêt, etc). Le scénario 2 considéré comme la reforestation, se focalise sur l'augmentation de la superficie de la forêt. 

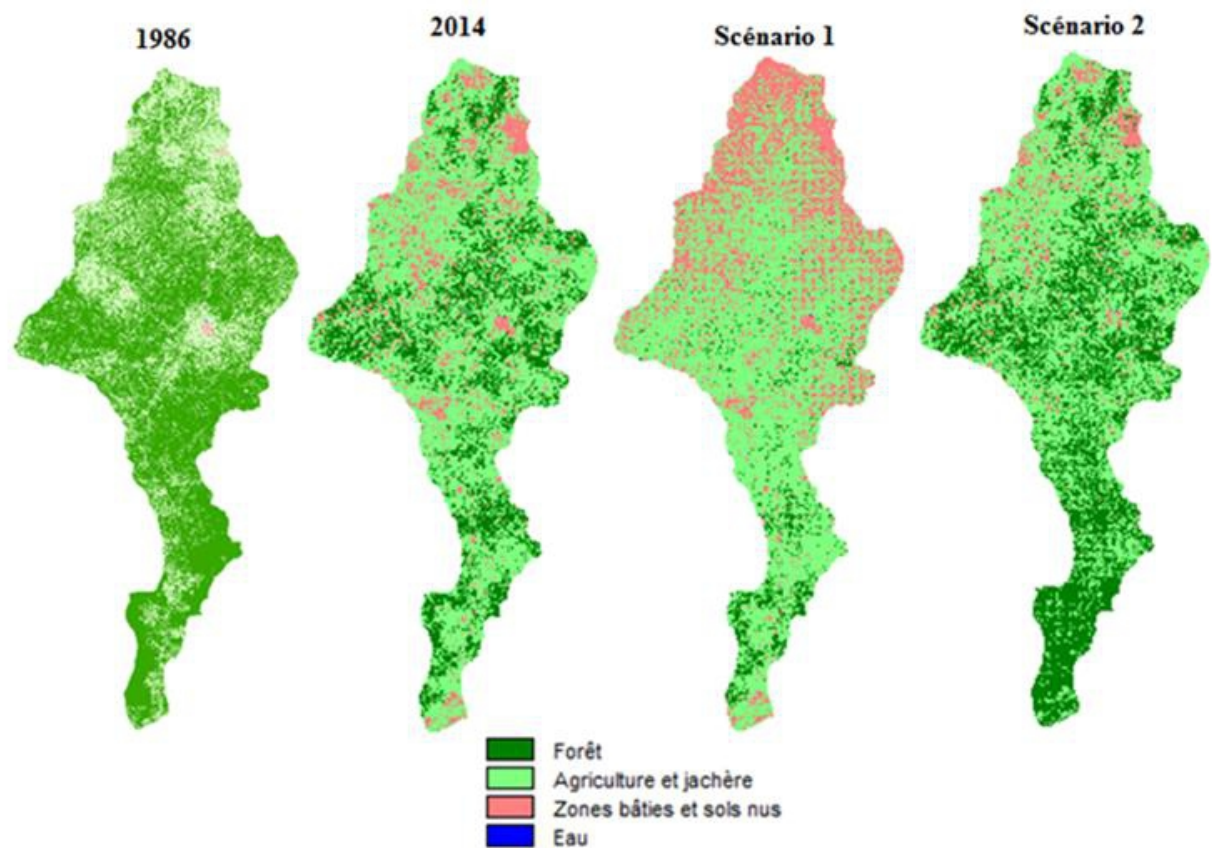

Figure 2. Scénarios d'occupation du sol basés sur les occupations du sol de 1986 et 2014

(Kouamé 2017)

Tableau 2 : Statistiques des classes d'occupation du sol en pourcentage (Kouamé 2017)

\begin{tabular}{ccccc}
\hline $\begin{array}{c}\text { Occupation } \\
\text { du sol }\end{array}$ & 1986 & 2014 & Scénario 1 & Scénario 2 \\
\hline $\begin{array}{c}\text { Forêt } \\
\text { Agriculture } \\
\text { et jachère }\end{array}$ & 65,60 & 25,70 & 6,56 & 37,90 \\
$\begin{array}{c}\text { Zones bâties } \\
\text { et sols nus }\end{array}$ & 1,03 & 62,13 & 66,82 & 54,71 \\
Eau & 0,01 & 12,15 & 26,60 & 7,32 \\
\hline
\end{tabular}

\section{Application du modèle CEQUEAU}

CEQUEAU est un modèle hydrologique distribué développé par l'INRSEAU (Morin et Parquet, 2007). L'évaluation de la performance des modèles hydrologiques passe par les processus de calage et de validation. Le calage et la validation ont été effectués en utilisant des débits. Trois années ont été utilisées pour le calage (1990-1992) et trois autres années ont été utilisées pour la validation (1993-1995) consistant à tester le modèle calé sur une série de données non utilisées dans l'étape du calage. Le coefficient de corrélation (R), le coefficient de Nash-Sutcliffe (NS) (1970) et les graphiques de comparaison des débits observés et simulés ont été utilisés pour évaluer la performance du modèle. Le coefficient de corrélation (R) et le coefficient de Nash-Sutcliffe (NS) (1970) sont généralement calculés (équation 1 et 2), ainsi : 
- le coefficient de corrélation (R)

$$
R=\frac{\sum_{i=i}^{n}\left(Q_{o b s, i}-\bar{Q}_{o b s}\right)\left(Q_{s i m, i}-\bar{Q}_{s i m}\right)}{\sqrt{\sum_{i=1}^{n}\left(Q_{o b s, i}-\bar{Q}_{o b s}\right)^{2} \sum_{i=1}^{n}\left(Q_{s i m, i}-\bar{Q}_{s i m}\right)^{2}}}
$$

La valeur de $\mathrm{R}$ indique la relation entre les débits observés et les débits simulés. La relation est parfaite lorsque $\mathrm{R}=1$. $\mathrm{R}<0,5$ est généralement décrite comme faible, comme aussi indiqué :

- le coefficient de Nash-Sutcliffe (NS) (1970).

$N S=1-\frac{\sum_{i=1}^{n}\left(Q_{o b s, i}-Q_{s i m, i}\right)^{2}}{\sum_{i=1}^{n}\left(Q_{o b s, i}-\bar{Q}_{o b s}\right)^{2}}$

$Q_{o b s, i}$ est le débit observé et $Q_{s i m, i}$ est le débit simulé pour le pas de temps i. $\bar{Q}_{o b s}$ et $\bar{Q}_{s i m}$ sont respectivement les moyennes des débits observés et simulés. $\mathrm{N}$ est le nombre d'observations.

Le coefficient de Nash-Sutcliffe prend des valeurs qui varient entre 1 et moins infini. Plus la valeur de Nash-Sutcliffe s'approche de l'unité, meilleure est la simulation.

L'évaluation des impacts des changements climatiques et des climatiques d'occupation du sol a été réalisée séparément. Pour évaluer les impacts des changements d'occupation du sol, les taux de forêt issus des scénarios 1 et 2 ont été intégrés dans le modèle CEQUEAU. Les résultats ont été analysés en termes de bilan hydrologique.

Concernant les impacts des changements climatiques sur les débits, les scénarios des changements climatiques générés sur deux périodes de 30 ans : 2040-2069 centrée sur 2050 et 2070-2099 centrée sur 2080 à l'aide de la méthode de changement de delta appelée aussi méthode des perturbations ou méthodes des anomalies (Minville et al., 2008 ; Montenegro et Ragab 2012 ; Juan et al., 2013; Fan et Shibata, 2015). Dans le cas des températures minimales et maximales, la différence (delta $\mathrm{T}$ ) entre les températures moyennes mensuelles interannuelles futures simulées d'un horizon et les températures moyennes mensuelles interannuelles simulées de la période de référence est additionnée aux données journalières historiques (Equation 3).

$T_{\text {scen }, j, h}=T_{o b s, j}+\left(\bar{T}_{\text {scen }, m, h}-\bar{T}_{\text {réf }, m}\right)$

Où :

- $T_{s c e n, j, h}$ est la température journalière de l'horizon considéré.

- $T_{o b s, j}$ est la température journalière observée.

- $\bar{T}_{s c e n, m, h}$ est la température moyenne mensuelle interannuelle de l'horizon considéré. 
- $\bar{T}_{\text {réf,m }}$ est la température moyenne mensuelle interannuelle de la période de référence.

- $\bar{T}_{\text {scen,m,h }}-\bar{T}_{\text {réf,m }}$ est Delta T.

Pour ce qui est des précipitations, c'est le rapport (delta $\frac{P}{P}$ ou ratio) des précipitations mensuelles simulées d'un horizon sur les précipitations totales mensuelles simulées de la période de référence qui est multiplié aux précipitations totales journalières observées de la période de référence (équation 4).

$P_{\text {scen }, j, h}=P_{o b s, j} \times\left(\frac{\bar{P}_{\text {scen }, m, h}}{\bar{P}_{\text {ré } f, m}}\right)$

Où :

- $\quad P_{s c e n, j, h}$ est la précipitation journalière de l'horizon considéré.

- $P_{o b s, j}$ est la précipitation journalière observée.

- $\bar{P}_{s c e n, m, h}$ est la précipitation moyenne mensuelle interannuelle de l'horizon considéré.

- $\bar{P}_{\text {réf,m }}$ est la précipitation moyenne mensuelle interannuelle de la période de référence.

- $\frac{\bar{P}_{\text {scen }, m ; h}}{\bar{P}_{\text {réf }, m}}$ est Delta $\frac{P}{P}$ ou $\Delta \mathrm{P}$ ou ratio.

Les séries météorologiques (températures journalières minimales et maximales, et les précipitations journalières) créées ont été incorporées dans le modèle CEQUEAU. Les résultats ont été analysés en termes de bilan hydrologique.

\section{Résultats}

\section{Calibration et validation du modèle CEQUEAU}

Les indices d'évaluation statistique de la performance de CEQUEAU sont résumés dans le tableau 3. L'évaluation de la performance du modèle a été faite sur la base journalière. Pour la calibration, les coefficients de Nash et de corrélation sont respectivement 0,65 et 0,68 . Le débit observé est $26,3 \mathrm{~m} 3 / \mathrm{s}$ et le débit calculé est $24,8 \mathrm{~m} 3 / \mathrm{s}$. Les valeurs journalières interannuelles de la lame d'eau observées et calculés sont respectivement 120,9 mm et 116,6 mm. Au niveau de la validation, sur la période 1993-1995, les coefficients de Nash et de corrélation sont respectivement 0,46 et 0,68 . Le débit observé est 31,1 $\mathrm{m}^{3} / \mathrm{s}$ et le débit calculé est $30,3 \mathrm{~m} 3 / \mathrm{s}$. Les valeurs journalières interannuelles de la lame d'eau observée et calculée sont respectivement 142,9 mm et 139,2 $\mathrm{mm}$. Sur l'autre période de validation 1990-1995, le coefficient de Nash est 0,69 et celui de la corrélation est 0,84 . Le débit observé est $27,8 \mathrm{~m} 3 / \mathrm{s}$ et le 
débit calculé est 26,6 m3/s. Les valeurs journalières interannuelles de la lame d'eau observée et calculée sont respectivement $127,7 \mathrm{~mm}$ et $122,1 \mathrm{~mm}$.

Tableau 3. Evaluation statistique de CEQUEAU pour les périodes de calibration et de validation.

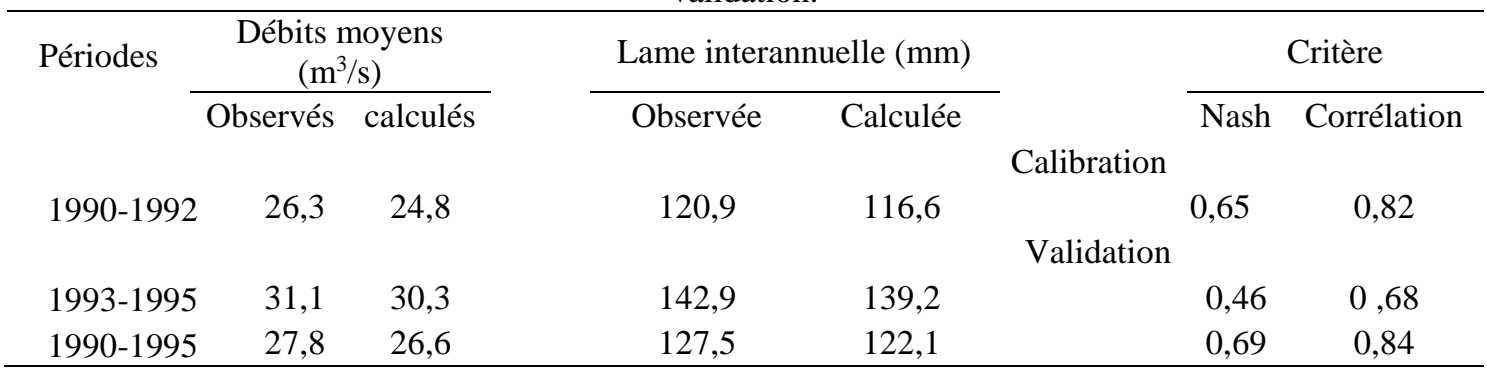

L'analyse des critères numériques renseigne d'une façon objective sur la précision globale des simulations. Il est cependant difficile d'apprécier les éventuelles différences entre les débits observés et calculés. Par contre, l'analyse des différents graphiques, même si elle peut être subjective, permet de percevoir les erreurs de simulation. Comme l'indique la figure 3, les débits calculés sont soustimés en janvier et de mai à août, et d'octobre à décembre. Le modèle surestime les débits calculés de février à avril et en septembre. Sur la figure 4, les débits calculés sont surestimés de décembre à mai et à août. Le modèle sousestime les débits calculés de juin à juillet et de septembre à novembre.

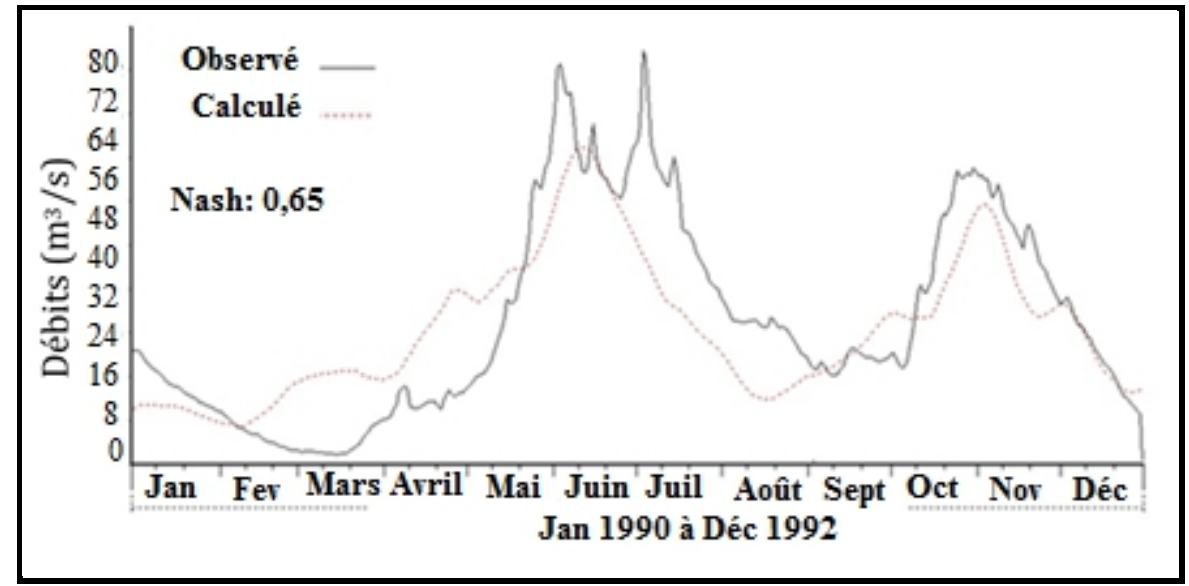

Figure 3. Débits journaliers interannuels observés et calculés pour la période de calage (1990-1992). 


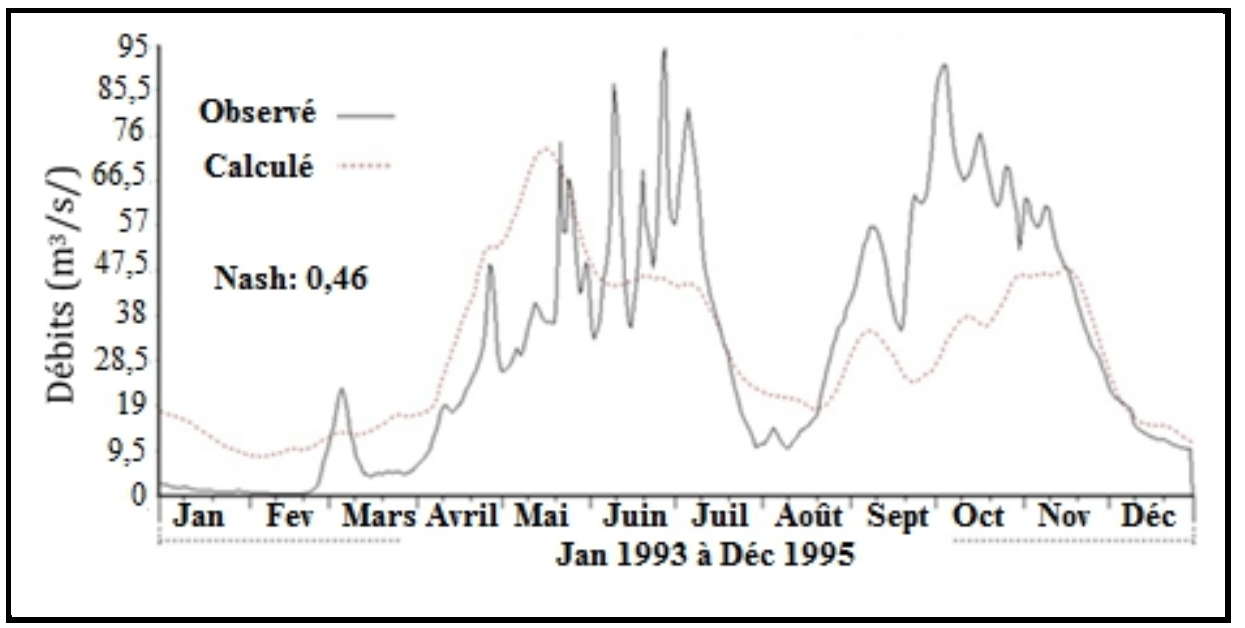

Figure 4. Débits journaliers interannuels observés et calculés pour la période de validation (1993-1995).

L'examen du graphique des débits cumulés (figure 5) sur la période complète indique une assez bonne similitude entre les débits observés et les débits calculés avec un critère de Nash de 0,95 .

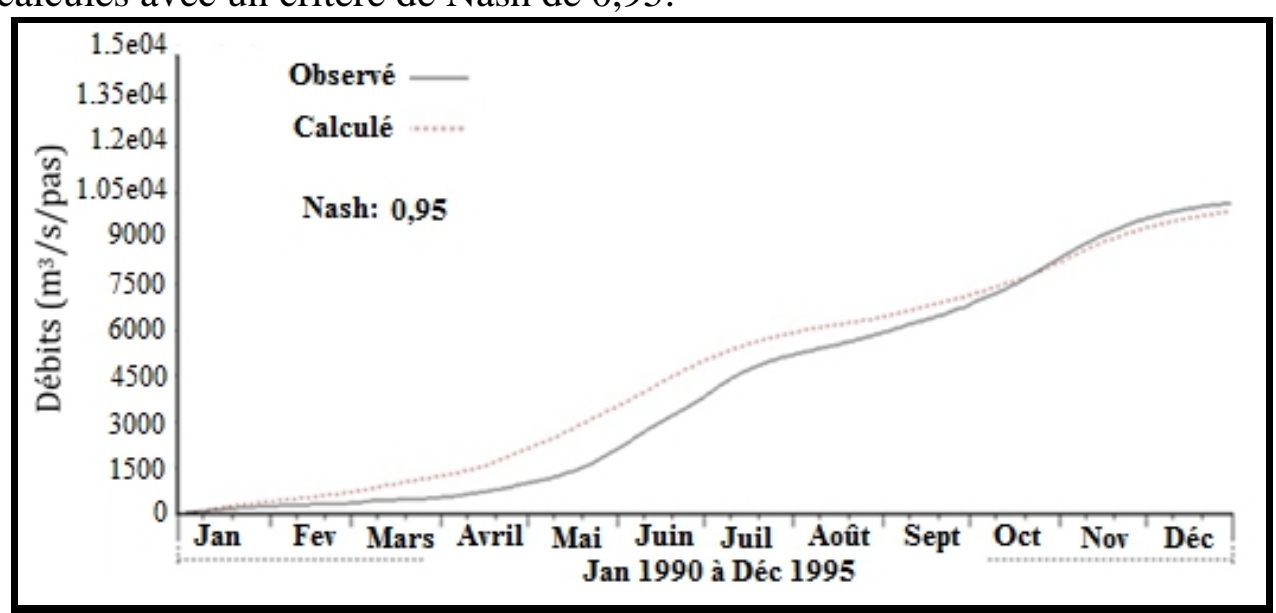

Figure 5. Débits mensuels cumulés observés et calculés pour la période 1990 à 1995.

Le graphique des débits moyens mensuels interannuels présenté avec le style histogramme (Figure 6), permet de comparer les moyennes mensuelles observées et calculées afin de s'assurer que les débits de tous les mois sont bien simulés. Le graphique de la figure 6 montre une surestimation des débits du mois de décembre à mai et une sous-estimation de juin à novembre. 


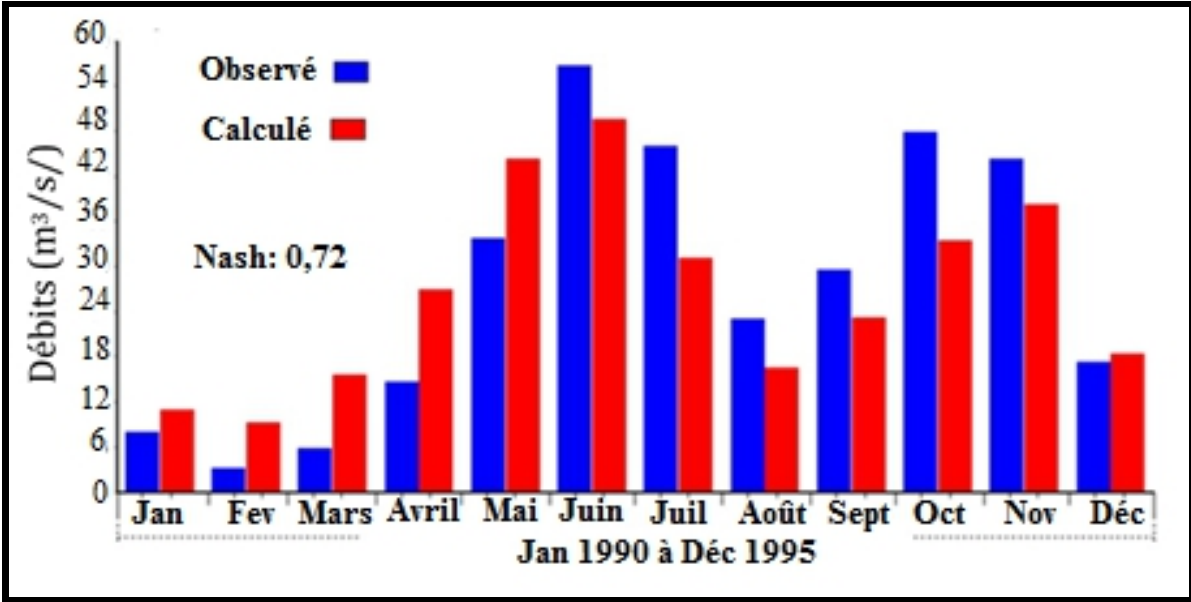

Figure 6. Débits moyens mensuels interannuels observés et calculés pour la période 1990 à 1995.

\section{Impacts des changements climatiques et des changements d'occupation du sol}

\section{Impacts des changements d'occupation du sol}

Les résultats des impacts sur les débits mensuels dûs aux changements d'occupation du sol des scénarios 1 et 2 par rapport à la période de référence (période d'observation) sont présentés à la figure 7. Les débits sur la période de référence et ceux issus du scénario 2 sont presque identiques sur tous les mois à l'exception des périodes des hautes eaux (mai à juillet et octobre à novembre). Sur ces périodes les débits issus du scénario 2 sont peu élevés par rapport à ceux de la période de référence. Les débits issus du scénario 1 sont plus élevés que les débits de la période de référence et ceux issus du scénario 2 sur tous les mois et sur les périodes des hautes eaux, leur élévation est encore plus accentuée. Les bilans hydrologiques issus des scénarios 1 et 2 sont présentés dans le tableau 4. A partir de la simulation sur la période de référence avec $4608,9 \mathrm{~km}^{2}$ de forêt, la lame d'eau précipitée de 1214,4 $\mathrm{mm}$ se repartie en lame d'eau évapotranspirée (1064, $8 \mathrm{~mm}$ ), en lame d'eau écoulée de 125,8 $\mathrm{mm}$ et une lame d'eau infiltrée de $23,8 \mathrm{~mm}$. Le bilan hydrologique établi à partir du scénario 1 avec $460,7 \mathrm{~km}^{2}$ de forêt (soit une diminution de $90 \%$ par rapport à la période de référence), est comme suit : une lame d'eau écoulée de $139,2 \mathrm{~mm}$ (soit une augmentation de 10,6 \% par rapport à la période de référence), une lame d'eau évapotranspirée de 1054,5 (soit une diminution de $1 \%$ par rapport à la période de référence), une lame d'eau infiltrée de 20,7 mm (soit une diminution de 13,1\% par rapport à la période de référence). En comparaison aux résultats de la période de référence, le scénario 2 avec une diminution de $42 \%$ de la surface forestière, indique une diminution de lame d'eau ruisselée de $1,7 \%$, une baisse des lames d'eau évapotranspirée et infiltrée respectivement de $0,1 \%$ et de $2,5 \%$. Le scénario 1 produit plus de 
débits alors que le scénario 2 produit en quantités réduites. Le scénario 2 donne une évapotranspiration plus élevée que le scénario 1.

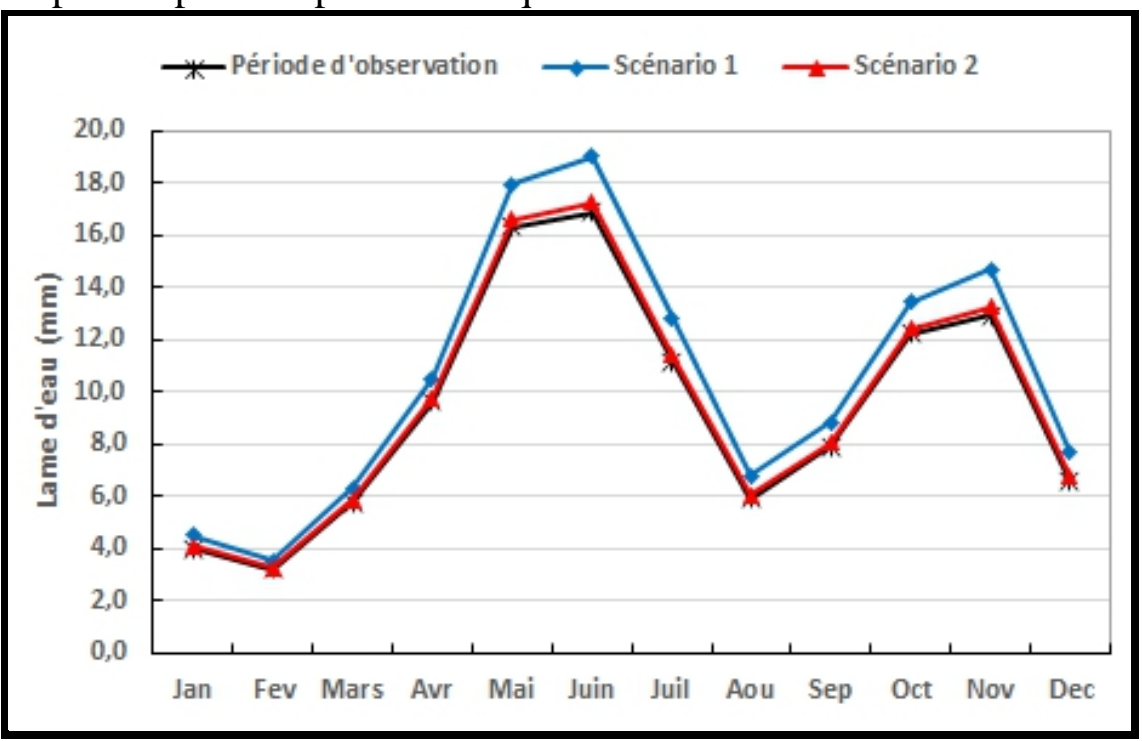

Figure 7. Comparaison des débits mensuels de la période de référence avec les débits issus des scénarios 1 et 2 .

Tableau 4. Comparaison des scénarios 1 et 2 par rapport à la période de référence. a) superficie d'occupation du sol, b) débits, évapotranspiration, infiltration. $\%$ de changements par

a) rapport à la période de référence

\begin{tabular}{|c|c|c|c|c|c|}
\hline & $\begin{array}{c}\text { Période de } \\
\text { référence }\end{array}$ & Scénario 1 & Scénario 2 & Scénario 1 & Scénario 2 \\
\hline Forêt & 4608,9 & 460,7 & 2665,3 & $-90,0$ & $-42,2$ \\
\hline $\begin{array}{l}\text { Agriculture } \\
\text { et jachère }\end{array}$ & 2342,5 & 4694,8 & 3844,1 & 100,4 & 64,1 \\
\hline $\begin{array}{l}\text { Zones bâties } \\
\text { et sols nus }\end{array}$ & 72,5 & 1868,3 & 514,2 & 2477,0 & 609,2 \\
\hline
\end{tabular}

b) référence

\begin{tabular}{ccccccc}
\hline & $\begin{array}{c}\text { Période de } \\
\text { référence }\end{array}$ & Scénario 1 & Scénario 2 & & Scénario 1 & Scénario 2 \\
\cline { 1 - 3 } \cline { 6 - 7 } Lame d'eau & 125,8 & 139,2 & 128,0 & & 10,6 & 1,7 \\
$(\mathrm{~mm})$ & 1064,8 & 1054,5 & 1063,2 & & $-1,0$ & $-0,1$ \\
ETR $(\mathrm{mm})$ & 23,8 & 20,7 & 23,2 & & $-13,0$ & $-2,5$ \\
$\begin{array}{c}\text { Infiltration } \\
(\mathrm{mm})\end{array}$ & 23,8 & & & &
\end{tabular}




\section{Impacts des changements climatiques}

La figure 8 présente les relations température-précipitation. L'augmentation des températures diminuerait les précipitations avec CSIRO sur les deux tranches de temps et à l'horizon 2050 avec GDFL sous le scénario A2. Cependant, cette augmentation entrainerait une augmentation des précipitations avec les modèles ECHAM 5 et HadCM3 sur les deux tranches de temps et avec le modèle GDFL sous le scénario B2.

Les lames d'eau moyennes mensuelles pour les conditions naturelles (période de référence) et simulées avec les données météorologiques pour les différents scénarios sont montrées à la figure 9 . Il y a une grande variation des lames d'eau mensuelles. La figure 10 présente les variations des débits moyens mensuels prévus sur les deux périodes futures par rapport à la période de référence. Avec le modèle GDFL, les lames d'eau diminueraient sur les deux horizons. Le modèle CSIRO indiquerait une diminution des lames d'eau au mois de mars sur les deux tranches de temps. Sous le scénario A2 et à l'horizon 2050, les lames connaitraient une augmentation de janvier à mars et aux mois de septembre et de décembre avec le modèle ECHAM5. A l'horizon 2080, les lames d'eau diminueraient aux mois de mars, de juin et d'octobre avec le même modèle et sous le même scénario. Sous le scénario B2, le modèle ECHAM5 indiquerait une augmentation des lames d'eau aux mois de janvier et de décembre à l'horizon 2050. A l'horizon 2080 et sous le scénario B2, le même modèle indiquerait une augmentation des lames d'eau de janvier à mars et de novembre à décembre. Avec le modèle HadCM3 et sous le scénario A2, les lames d'eau connaitraient une diminution de septembre à novembre à l'horizon 2050 et une diminution aux mois de septembre et d'octobre à l'horizon 2080. Sous le scénario B2, le même modèle indiquerait une diminution des lames d'eau aux mois de septembre et d'octobre sur les deux tranches de temps.

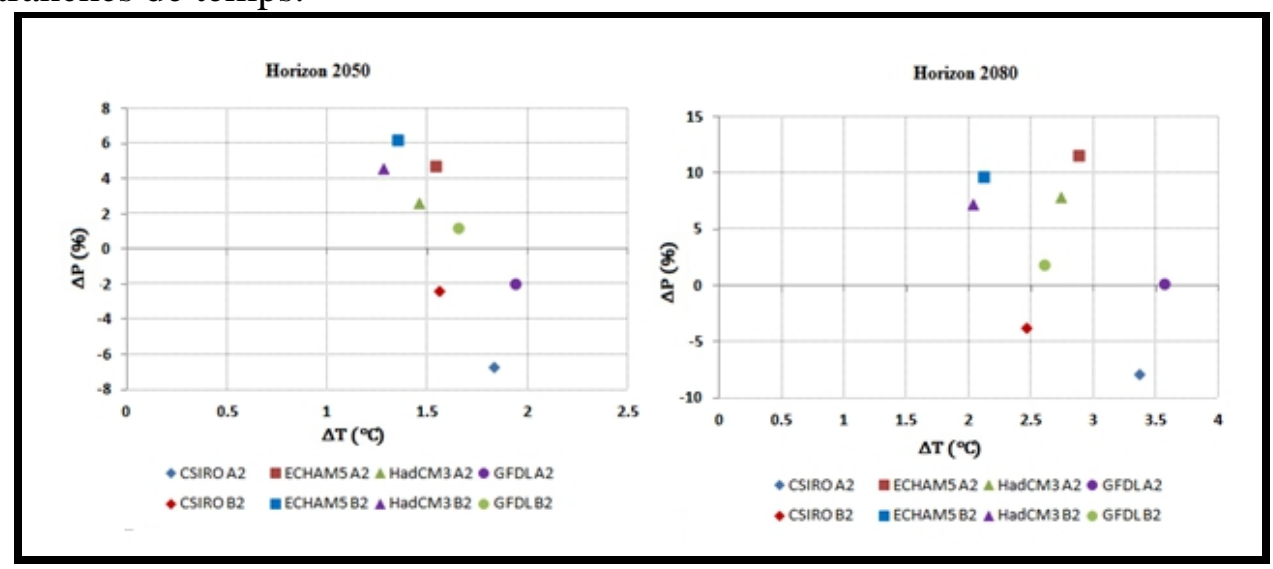

Figure 8. Diagramme de dispersion montrant les relations précipitations-température; 


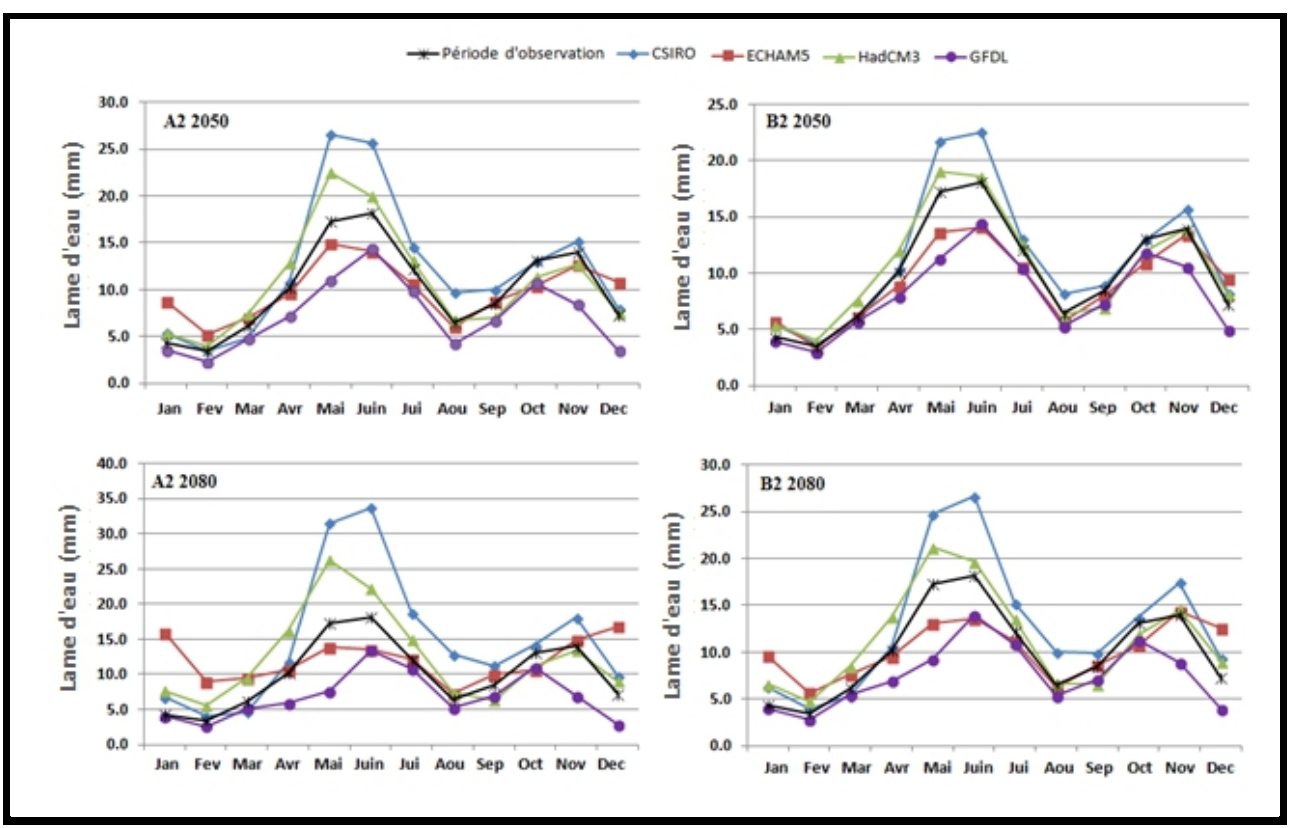

Figure 9. Evolution des lames d'eau mensuelles sur les différentes périodes.

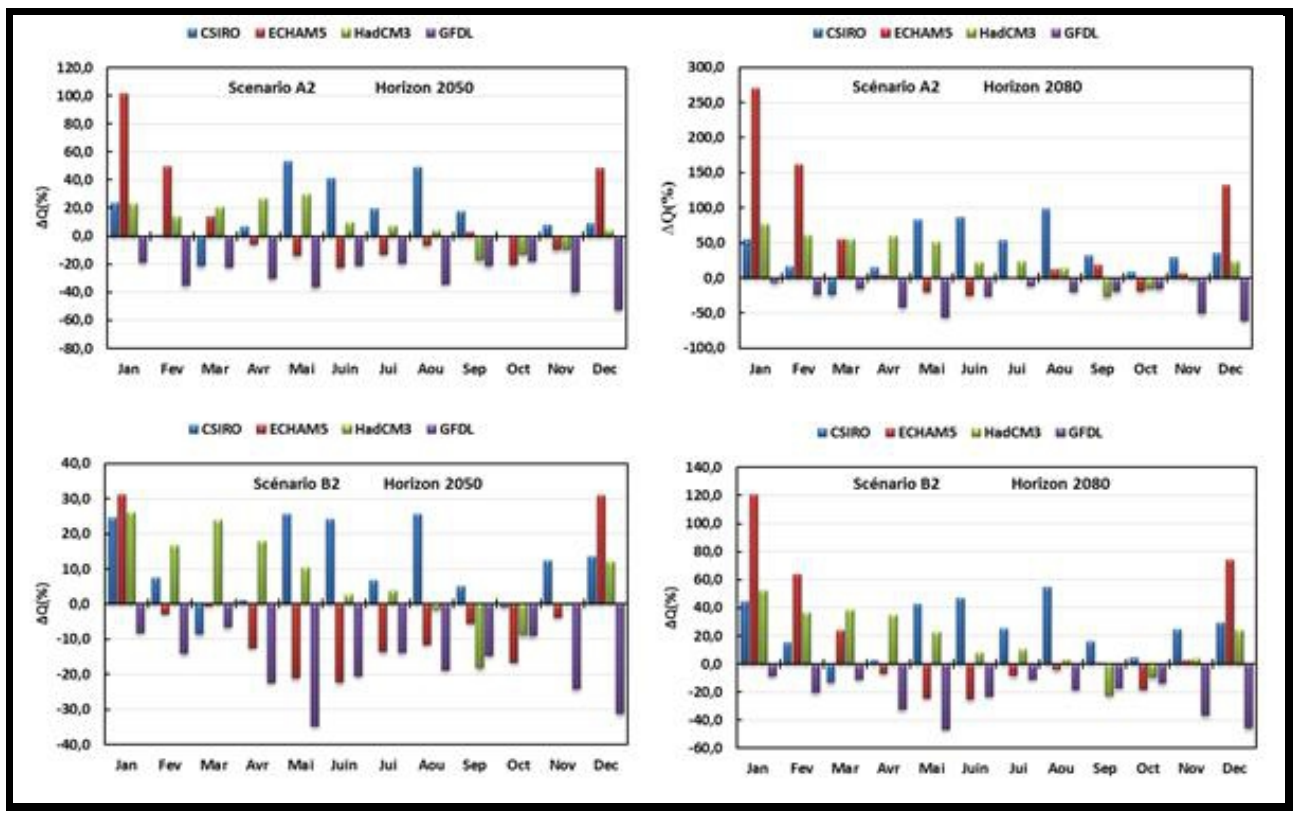

Figure 10. Variations (\%) des débits moyens mensuels prévus sur les deux périodes futures par rapport à la période de référence.

Dans le même ordre d'idée que les lames d'eau mensuelles, la figure 11 présente les variations des lames d'eau annuelles. Sous les deux scénarios et sur les deux tranches de temps, les modèles CSIRO et HadCM3 indiqueraient une augmentation des lames d'eau annuelles. Avec le modèle 
CSIRO, les lames d'eau connaitraient une augmentation de $21 \%$ et de $13 \%$ respectivement sous le scénario A2 et sous le scénario B2 à l'horizon 2050. A l'horizon 2080 et avec le même modèle, les lames d'eau augmenteraient de 47 $\%$ sous le scénario A2 et de $26 \%$ sous le scénario B2. Pour ce qui est du modèle HadCM3 et à l'horizon 2050, les lames d'eau annuelles augmenteraient de $8 \%$ sous le scénario A2 et de $5 \%$ sous le scénario B2. A l'horizon 2080 et avec le même modèle, les lames connaitraient une augmentation de $24 \%$ et de $13 \%$ respectivement sous le scénario A2 et sous le scénario B2. Avec le modèle ECHAM5, les lames d'eau diminueraient de $2 \%$ et de $9 \%$ respectivement sous le scénario A2 et sous le scénario B2. Cependant, elles augmenteraient de $20 \%$ sous le scénario A2 et de $1 \%$ sous le scénario B2 avec le même modèle. Le modèle GDFL indiquerait une diminution des lames d'eau sur les deux horizons et sous les deux scénarios. Elles diminueraient donc de $29 \%$ et de $20 \%$ respectivement sous le scénario A2 et sous le scénario B2 à l'horizon 2050 et de $32 \%$ sous le scénario A2 et de $26 \%$ sous le scénario B2 à l'horizon 2080. Avec l'ensemble multimodèle, les lames d'eau connaitraient une diminution de $0,5 \%$ et de $2,75 \%$ respectivement sous les scénarios A2 et B2 à l'horizon 2050. A l'horizon 2080, l'ensemble multimodèle indiquerait une augmentation des lames d'eau de $14,75 \%$ sous le scénario A2 et de 3,5\% sous le scénario B2. Les bilans hydrologiques issus des scénarios A2 et B2 sont présentés dans le tableau 5. Les valeurs annuelles de l'évapotranspiration n'excèdent pas celles des précipitations et les valeurs de l'infiltration sont inférieures à.la valeur de la période de référence.

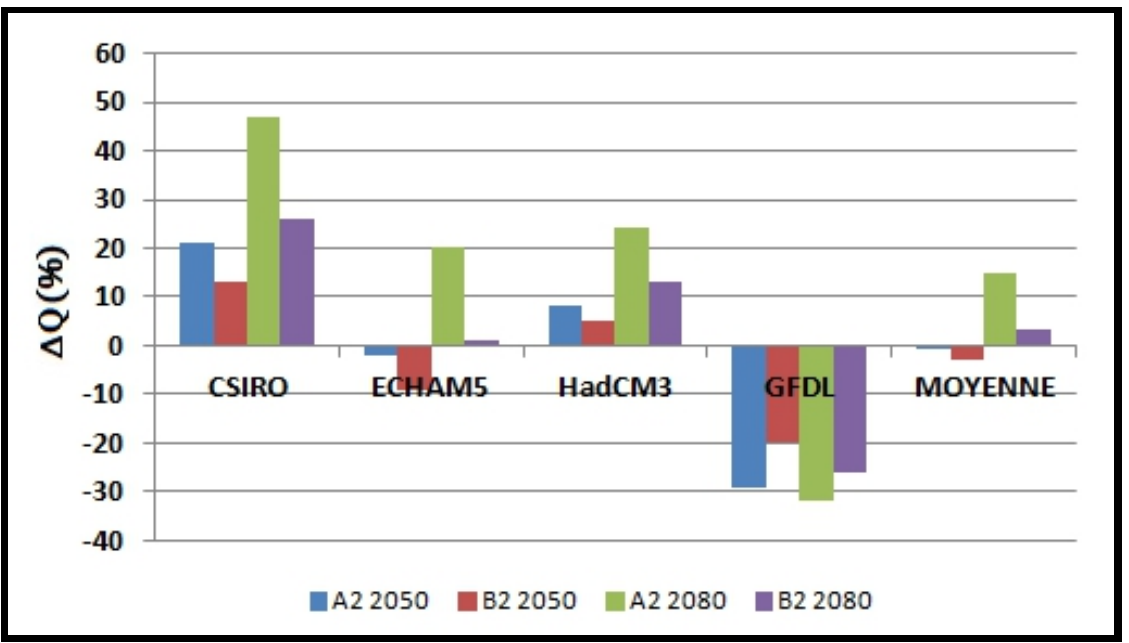

Figure 11. Variations des débits moyens annuels prévus sur les deux périodes futures par rapport à la période de référence. 
Tableau 5. Bilan hydrologique pour les scénarios A2 et B2 (Période de référence : Pluie $(1214,4)$ lame $(125,8 \mathrm{~mm})$ et ETR $(1064,8 \mathrm{~mm})$ Infiltration $(23,8 \mathrm{~mm})$

\begin{tabular}{cccccc}
\hline Scénarios & MCG & Pluie $(\mathrm{mm})$ & Lame $(\mathrm{mm})$ & ETR (mm) & $\begin{array}{c}\text { Infiltration } \\
(\mathrm{mm})\end{array}$ \\
\hline \multirow{4}{*}{ A2 2050 } & CSIRO & 1115 & 147 & 960 & 8 \\
& ECHAM5 & 1155 & 119 & 1041 & -5 \\
& GFDL & 1037 & 86 & 945 & 6 \\
& HadCM3 & 1197 & 130 & 1075 & -8 \\
\hline \multirow{5}{*}{ B2 2050 } & CSIRO & 1126 & 136 & 986 & 4 \\
& ECHAM5 & 1131 & 110 & 1025 & -4 \\
& GFDL & 1063 & 97 & 963 & 3 \\
A2 2080 & HadCM3 & 1186 & 127 & 1069 & -10 \\
& CSIRO & 1126 & 177 & 941 & 8 \\
& ECHAM5 & 1192 & 144 & 1068 & -20 \\
& GFDL & 993 & 83 & 904 & 6 \\
H2 2080 & HadCM3 & 1273 & 149 & 1138 & -14 \\
& CSIRO & 1130 & 153 & 972 & 5 \\
& ECHAM5 & 1171 & 123 & 1053 & -5 \\
& GFDL & 1033 & 90 & 941 & 2 \\
\hline & HadCM3 & 1234 & 136 & 1109 & -11 \\
\hline
\end{tabular}

\section{Discussion}

La vérification de la performance du modèle CEQUEAU dans une perspective d'évaluation des impacts des changements climatiques et des changements d'occupation du sol sur le bassin versant de la rivière Davo a conduit dans l'ensemble à des résultats satisfaisants, car, la performance du modèle a été vérifiée par plusieurs indices. Les débits calculés sont dans des proportions acceptables. Les résultats de la calibration et de la validation sont conformes aux résultats trouvés par Brou (2002) sur le bassin versant de Sassandra à Soubré et par Kouadio (2011) sur les bassins versants d'Agneby et de Boubo. Ces deux auteurs ont appliqué le modèle CEQUEAU. Les résultats de la vérification du modèle CEQUEAU sont également similaires aux résultats de Kouakou (2011) qui a utilisé le modèle GR2M sur le bassin versant de Comoé. Toutefois, il y a quelques incertitudes dans l'exercice de la calibration et de la validation du modèle CEQUEAU. En effet, la qualité des résultats dépend des données d'entrée, des paramètres et de la structure du modèle. CEQUEAU a besoin des données physiographiques et hydrométéorologiques (pluie, température et débit) pour son fonctionnement. Le processus de la mise en place de ces données est une tâche complexe et difficile. Pour avoir des données physiographiques fiables et de bonne qualité, il faut une connaissance approfondie de la zone d'étude. Ce qui n'a toujours pas été le cas. Parmi les données hydrométéorologiques pour le fonctionnement de CEQUEAU, les données de débits observés pourraient être à l'origine des écarts constatés sur les périodes de calibration et de validation. 
Ces mêmes constats ont été faits par Goula et al., (2009). En effet, leur mesure nécessite dans un premier temps que le cours d'eau soit taré par l'établissement de la courbe débits-hauteurs limnimétriques. Ensuite, à partir de cette courbe de tarage, les hauteurs limnimétriques journalières relevées sont ainsi traduites en données de débits (Kouadio, 2011). Concernant les autres données hydrométéorologiques à savoir les données météorologiques, la température reste précise, car, elle varie très peu dans l'espace. Considérée comme l'entrée principale des modèles pluie-débit, la pluie doit sa qualité aux caractéristiques des dynamiques météorologiques sur le bassin, aux pas de temps d'observation et à la densité de la répartition du réseau pluviométrique. De fortes incertitudes demeurent ainsi dans le protocole de collecte de ces données en terme de qualité, mais aussi en terme de quantité c'est-à-dire dans l'estimation de la précision spatiale nécessaire à ces mesures pour avoir une estimation fiable des quantités moyennes précipités au sein du bassin versant. Pour le modèle CEQUEAU, on s'intéresse aux quantités de pluie au niveau de la maille de discrétisation. Afin de représenter correctement la pluie sur chaque maille, deux méthodes d'extrapolation sont utilisées à savoir la méthode de polygone de Thiessen et la méthode de pondération des trois stations les plus proches. Les estimations des précipitations à l'aide de ces différentes méthodes peuvent être exactes à l'échelle du bassin, mais elles ne produisent pas des estimations précises des précipitations pour la modélisation hydrologique distribuée (Lopes, 1996). La difficulté pour représenter correctement la pluie vient essentiellement du fait que c'est un phénomène à la fois non continu dans le temps et variable dans l'espace. La variabilité spatiale de la pluie est une source majeure d'incertitude dans la modélisation hydrologique (Lopes, 1996 ; Elena et al., 2012).

Les effets des changements climatiques et des changements d'occupation du sol sur l'hydrologie ont été analysés séparément. Concernant les impacts des changements d'occupation du sol, le scénario 1 (déforestation) produit plus de débit que le scénario 2 (reforestation). Le scénario 2 donne une évapotranspiration plus élevée que le scénario 1. Ceci est du à plus de couverture végétale au niveau du scénario 2. Ainsi, l'augmentation des débits pourrait être attribuée à la diminution de l'évapotranspiration. Cette diminution de l'évapotranspiration pourrait être attribuée à la réduction de l'interception et de la transpiration. La forêt a des effets de réduire les débits. Les études de Githui (2008), Montenegro et Ragab (2012) ont indiqué une observation similaire où les débits sont réduits comme les résultats de la reforestation. Les effets des changements d'occupation du sol ont peu d'influence sur l'hydrologie du bassin versant. Ce constat est similaire à d'autres études (Kim et al., 2013 ; Suzana et Ragad, 2012). Suzana et Ragab, 2012 ont utilisé un modèle distribué et deux scénarios des changements d'occupation du sol pour simuler leurs impacts sur le cycle de l'eau. Les 
auteurs ont trouvé un effet minime des impacts des changements d'occupation du sol dus aux effets compensateurs dans un bassin complexe.

Quant aux impacts des changements climatiques sur l'hydrologie du bassin, ils ont de l'influence importante comparativement aux effets de l'occupation du sol.. Ces résultats sont semblables aux résultats de Kim et al., 2013. Toutefois, les résultats des impacts des changements climatiques dépendent des modèles climatiques, des scénarios d'émission et des périodes. Ces résultats sont similaires aux résultats de Minville et al. (2008), Forbes et al. (2011) et Juan et al. (2013). Les résultats indiquent qu'il existe des incertitudes dans les débits futurs. Sur le bassin versant, il y a eu l'usage d'un modèle hydrologique, d'une méthode de désagrégation, quatre modèles du climat, deux scénarios SRES et deux périodes de calcul. L'ensemble des croisements possibles définit un total de seize cas. Chaque étape de l'ensemble est soumise à des incertitudes. De toutes ces incertitudes possibles, celle entrainée par le choix d'un modèle climatique est le plus important (Kay et al., 2009; Prudhomme et al., 2009 ; Chen et al., 2011). Ainsi, une étude des impacts basée sur l'usage d'un modèle climatique devrait être interprétée avec beaucoup de soin.

\section{Conclusion}

Cette étude s'est intéressée aux impacts des changements climatiques et des changements d'occupation du sol sur les ressources en eau dans le bassin versant de la rivière Davo en utilisant le modèle hydrologique distribué CEQUEAU. En dépit des incertitudes, les évaluations statistiques et graphiques sur la performance du modèle CEQUEAU ont montré qu'il pouvait être utilisé pour évaluer les impacts des changements climatiques et des changements d'occupation du sol. L'évaluation des impacts des changements de l'occupation-utilisation du sol et changements d'occupation du sol a été réalisée séparément. Le scénario 1 produit plus de débit que le scénario 2 . En d'autres termes, le scénario 2 donne en moyenne plus d'évapotranspiration que le scénario 1 . Ceci est dû à plus de couverture végétale au niveau du scénario 2. Quant aux impacts des changements climatiques, Il n'y a pas de consensus entre les quatre modèles climatiques. Les résultats des impacts des changements climatiques sont incertains et dépendent des modèles climatiques, des scénarios d'émission et des périodes.

Dans une perspective de mettre en place des stratégies d'adaptation et d'atténuation, il convient d'utiliser des scénarios RCP (Representative Concentration Pathways), d'appliquer les modèles climatiques régionaux et faire l'évaluation combinée des impacts des changements climatiques et des changements d'occupation du sol.

En recommandation, pour lutter contre la désertification du bassin versant de la rivière Davo, les décideurs politiques doivent considérer le scénario 2 de 
l'occupation du sol qui se focalise sur l'augmentation de la superficie de la forêt.

\section{References:}

1. Boyd E., Corbera E., Estrada M., 2008. UNFCCC negotiations (preKyoto to COP-9): what the process says about the politics of CDMsinks. International environmental agreements politics. Law and Economics, 8(2): 1-18.

2. ecommde Sassandra (Côte d'Ivoire). Thèse de doctorat $3^{\text {éme }}$ cycle, Université d'Abobo-Adjamé. 146 p.

3. Brou, Y. T, 2010. Variabilité climatique, déforestation et dynamique agrodémographique en Côte d'Ivoire. Sécheresse, 21 : 1-6.

4. Brou Y. T., 2005. Climat, mutations socio-économiques et paysages en Côte d'Ivoire. Mémoire de synthèse des activités scientifiques présentées en vue de l'obtention de l'Habilitation à diriger des recherches. Université des sciences et technologies de Lille, 212 p.

5. Chen J., Brissette F. P., Poulin A., Leconte R., 2011: Overall uncertainty study of the hydrological impacts of climatechange for a Canadian watershed.Water Resources Research, 47, W12509, 16 p.

6. Cornelissen T., Diekkrüge B., Giertz S., 2013. A comparison of hydrological models for assessing the impact of land use and climate change on discharge in a tropical catchment. Journal of Hydrology, 498: 221-236.

7. Eastman J.R. 2005. IDRISI Andes, Guide to GIS and Image Processing. Clark University, Worcester: 87-131.

8. Elena V., Michele D. L., Aldo F., 2012. A simplified framework for assessing the impact of rainfall spatial variability on the hydrologic response. Advances in Water Resources, 46: 1-10.

9. Fan M., Shibata H., 2015. Simulation of watershed hydrology and stream water quality under land use and climate change scenarios in Teshio River watershed, northern Japan. Ecological Indicators 50: 7989.

10. Forbes K.A., Kienzle S.W., Coburn C.A., Byrne J.M., Rasmussen J., 2010. Modelling the impacts of selected GCM derived climate scenarios on the future hydrology of a hybrid watershed in the Oldman River watershed, Alberta, Canada. Climatic Change, 105 (3) : 555576.

11. Githiu W.F., 2008. Assessing the impacts of environmental change on the hydrology of the Nzoia Catchment, in the Lake Victoria basin. Thesis of doctor Engineering, Vrije Universiteit Brussel, 189 p.

12. Goula B.T.A., Kouadio Z.A., Kouakou K.E., N'goh Y.A., N'doume C., Savane I., 2009. Simulation du comportement hydrologique du 
bassin versant de l'Agneby, en Côte d'Ivoire. Revue Ivoirienne des Sciences et Technologie, 13 : 91-119.

13. Goula B.T.A., Savane I., Konan B., Fadika V., Kouadio G.B., 2006. Impact de la variabilité climatique sur les ressources hydriques des bassins de N'zo et N'zi en Côte d'Ivoire (Afrique tropicale humide).Vertigo, $1: 1-12$.

14. Juan M.B., Walter C., Adriano R.D.P., Daniel A., Federico D., 2013. Impact of projected climate change on hydrologic regime of the Upper Paraguay River Basin. Climatic

15. Kay A., Davies H., Bell V., Jones R., 2009. Comparison of uncertainty sources for climate change impacts: flood frequency in England. Climatic Change, 92: 41-63.

16. Kim J., Choi J., Choi C., Park S., 2013. Impacts of changes in climate and land use/land cover under IPCC RCP scenarios on streamflow in the Hoeya River Basin, Korea. Science of the Total Environment, 452453: 181-195.

17. Kouadio Z.A., 2011. Dynamique de l'occupation du sol et comportement hydrologique : cas des basssins versants côtiers de l'Agnéby et du Boubo (Côte d'Ivoire). Thèse unique de doctorat, Universitéd'Abobo-Adjamé, 188 p.

18. Kouakou K.E., 2011. Impacts de la variabilité climatique et du changement sur les ressources en eau en Afrique de l'Ouest : Cas du bassin versant de la Comoé. Thèse unique de doctorat, Université d'Abobo-Adjamé, $170 \mathrm{p}$.

19. Kouakou K. E., Goula B. T. A., Savané I., 2007. Impacts de la variabilité climatique sur les ressources en eau de surface en zone tropicale humide : Cas du bassin versant transfrontalier de la Comoé (Côte d'Ivoire - Burkina Faso). European Journal of Scientific Research, 16 (1): 31-43.

20. Kouakou K.E., Goula B.T.A., Kouassi A.M., 2012. Analyze of climate variability and change impacts on hydro-climate parameters: case study of Côte d'Ivoire. International Journal of Scientific \& Engineering Research, 3 (2): 8 p.

21. Kouamé Y. M., 2017. Impacts des changements climatiques et de la dynamique de l'occupation du sol sur les eaux de surface dans le bassin versant de la Davo, Côte d'Ivoire. Thèse unique de doctorat, Université Nangui Abrogoua, $196 \mathrm{p}$.

22. Kouamé Y. M., Soro G. E., Kouakou K. E, Kouadio Z. A., Mélèdje N. H., Goula B. T., A., Savané I., 2014. Scénarios des changements climatiques pour les précipitations et les températures en Afrique subsaharienne tropicale humide cas du bassin versant de Davo, cote d'ivoire. Larhyss Journal, 18 : 197-213. 
23. Lopez, V. L., 1996. On the effect of uncertainty in spatial distribution of rainfall on catchment modeling. Catena, 28: 107-119.

24. Minville M., Brissette F., Leconte R., 2008. Uncertainty of the impact of climate change on the hydrology of a nordic watershed. Journal of Hydrology, 358: 70-83.

25. Montenegro S., Ragab R., 2012. Impact of possible climate and land use changes in the semi-arid regions: A case study from North Eastern Brazil. Journal of hydrology, 434-435: 55-68.

26. Morin G., Paquet P., 2007. Modèle hydrologique CEQUEAU, INRSETE. Rapport de recherche no R000926, 458 p.

27. Nash J.E., Sutcliffe J.V., 1970. River flow forecasting through conceptual models 1: a discussion of principles. Journal of Hydrology, 10(3): 282-290.

28. Neupane R. P., White J. D., Alexander S. E., 2015. Projected hydrologic changes in monsoon-dominated Himalaya Mountain basins with changing climate and deforestation. Journal of Hydrology, 525: 216-230.

29. Pielke L.A., 2005. Land use and climate change. Science, 310 : 16251626.

30. Praskievicz S, Chang H., 2011. Impacts of climate change and urban development on water resources in the Tualatin River Basin, Oregon. annals of the association of American geographers;101:249-71.

31. Prudhomme C., Davies H., 2009. Assessing uncertainties in climate change impact analyses on the river flow regimes in the UK. Part 1: baseline climate. Climatic Change, 93 (1-2): 177-195.

32. Savané I., Coulibaly K. M. et Gioan P., 2001. Variabilité climatique et ressources en eaux souterraines dans la région sémi-montagneuse de Man. Sécheresse, 12 (4): 231-237

33. Toua K.M., 1993. Economie de plantation et organisation de l'espace du Sud-ouest ivoirien. Thèse de Doctorat de 3ème cycle. Université Nationale de Côte d'Ivoire, $495 \mathrm{p}$

34. Yao A.B., Goula B.T.A., Kouadio Z.A., Kouakou K.E., Kante A. Sambou S., 2012: Analyse de la variabilité climatique et quantification des ressources en eau en zone tropicale humide : cas du bassin versant de la Lobo au Centre-Ouest de la Côte d'Ivoire. Revue Ivoirienne des Sciences et Technologie, 19 : 136-157.

35. Wigley T.M.L., 2008. MAGICC/SCENGEN 5.3: user manual (version 2), National Center for Atmospheric Research, Colorado, 81 p. 